\title{
Study the Effect of Thermal Gradient on Transverse Vibration of Non-Homogeneous Orthotropic Trapezoidal Plate of Parabolically Varying Thickness
}

\author{
Arun K. Gupta, Shanu Sharma \\ Department of Mathematics, M S College, Saharanpur, India \\ E-mail: gupta_arunnitin@yahoo.co.in, shanu.sharma1987@gmail.com \\ Received September 15, 2010; revised October 12, 2010; accepted October 15, 2010
}

\begin{abstract}
The present paper deals with the effect of linearly temperature on transverse vibration of non-homogeneous orthotropic trapezoidal plate of parabolically varying thickness. The deflection function is defined by the product of the equations of the prescribed continuous piecewise boundary shape. The non homogeneity of the plate is characterized by taking linear variation of the Young's modulus and parabolically variation of the density of the material. The non homogeneity is assumed to arise due to the variation in the density of the plate material and it is taken as parabolically. Rayleigh Ritz method is used to evaluate the fundamental frequencies. The equations of motion, governing the transverse vibrations of orthotropic trapezoidal plates, are derived with boundary condition clamped-simply supported-clamped-simply supported. Frequencies corresponding to first two modes of vibration are calculated for the trapezoidal plate for various combinations of the parameters of the non-homogeneity, thermal gradient, taper constant and for different values of the aspect ratios and shown by figures. All The results presented here are entirely new and are not found elsewhere. Comparison can only be made for homogeneous plates, and in that cases the results have been compared with those found in the existing literatures and are in excellent agreement.
\end{abstract}

Keywords: Thermal Gradient, Vibration, Orthotropic Trapezoidal Plate, Parabolically Thickness, Non-Homogeneity

\section{Introduction}

Plate structures are fundamental elements in engineering and are used in a variety of structural applications. Structures like aircraft wings, satellites, ships, steel bridges, sea platforms, helicopter rotor blades, space craft antennae and subsystems of more complex structures can be modeled as orthotropic plate elements. The practical importance of plates has made vibration analysis essential for avoiding resonance excited by internal or external forces. Plate structures undergoing transverse deflection can be classified into numerous regimes that describe the nature of their behavior and thus the characteristics of the mathematical problem. Plate theory has been applied to reduce vibration and noise in structures since the end of the $19^{\text {th }}$ century where it began with the work of German physicist Chladni, who discovered various modes of free vibrations experimentally. Since then it has developed into an escalating and expansive field with a wide variety of theoretical and empirical techniques, dealing with increasingly complicated problems. Also in recent years, the development of solid propellant rocket motors, the increased use of soft filaments in aerospace structure, high speed runways, many practical solid structure interaction problems such as floor slabs of multistory buildings or buildings activities in cold regions has intensified the need for solution of various problems of plates and other structure supported on elastic media.

In addition, the non-homogeneity of longitudinal to transverse modules of these new materials demands improvement in the existing analytical tools. As a result, the analysis of plate's vibrations has attracted many research workers, and has been considerably improved to achieve realistic results. In the design of modern high-speed aircraft and missile structures, swept wing and tail surfaces are extensively employed. The literature on the free vibrations of plates is vast. A number of researchers have 
worked on free vibration analysis of rectangular, circular, elliptical etc. plates and variable thickness. Little research work has been done on non uniform thickness trapezoidal plates as compared to the other plates.

Trapezoidal plates are used commonly as structural components in many engineering applications such as ships, aircraft, engineering constructions etc. many researcher have investigated the free vibration behavior of trapezoidal plates. Leissa's monograph [1-3] contains an excellent discussion of the subject of vibrating plates. Chopra and Durvasula [4] solved the problem of vibration of simple-supported trapezoidal plate symmetric trapezoids. Vibration of skew plate was discussed by Nair and Durvasula [5]. Thermal effect on axisymmetric vibration of an orthotropic circular plate of variable thickness was discussed by Tomar and Gupta [6]. Tomar and Gupta [7] solved the problem of vibration of orthotropic rectangular plate of linearly varying thickness with thermal gradient effect. Orris and Petyt [8] study of the vibration of trapezoidal plate by using finite element method. Narita, Maruyama and Sonada [9] studied the transverse vibration of clamped trapezoidal plate having rectangular orthotropy. Mirza and Bijlani [10] discussed the vibration of triangular plates. Bhatnagar and Gupta [11] solved the problem of thermal effect on vibration of visco elastic elliptic plate of variable thickness. Transverse free vibration of fully clamped symmetrical trapezoidal plates was discussed by Sahba [12]. Laura, Gutierrez and Bhat [13] studied the transverse vibrations of a trapezoidal cantilever plate of variable thickness. Liew and Lam [14] studied a Rayleigh-Ritz approach to transverse vibration of isotropic and anisotropic trapezoidal plates using orthogonal plate functions. Problem of variable of symmetric laminated cantilever trapezoidal composite plates was solved by Liew [15].Liew and Lim [16] studied the transverse vibration of trapezoidal plates of variable thickness: symmetric trapezoids. Qatu, Jaber and Leissa [17] work out to analyze the natural frequencies for completely free trapezoidal plates. Vibration of pretwisted cantilever trapezoidal symmetric laminates was discussed by Lim and Liew [18]. Sakiyama and Hung [19] studied the free vibration analysis of right triangular plates with variable thickness. Lal [20] studied the transverse vibration of orthotropic non uniform rectangular plate with continuously varying density. Leissa [21] discussed the historical bases of the Rayleigh and Ritz methods. Chi-Hung, Chien and Yen-Kuang [22] did experimental and numerical investigations for the free vibration of cantilever trapezoidal plates. Gupta, Johri and Vats $[23,24]$ work out to investigate the thermal effect on vibration of non homogeneous orthotropic rectangular plate having bi-directional varying thickness. Karami, Shahpari and Malekzadeh [25] have applied Differential
Quadrature Method (DQM) for static, free vibration, and stability analysis of skewed and trapezoidal composite thin plates without hole. Recently, Gupta and Sharma [26] solved the problem of thermally induced vibration of orthotropic trapezoidal plate of linearly varying thickness.

From the review of available literature it is observed that the linearly temperature on transverse vibration of non-homogeneous orthotropic trapezoidal plate of parabolically varying thickness with non-homogeneity effect has not been studied. So it is necessary to analyze this kind of problem using elasticity theory based Rayleigh Ritz method to evaluate for the most accurate behavior of frequencies of the plate. Therefore, a method is developed to study the problem both theoretically and experimentally. In order to calculate natural frequencies for first and second mode of vibration, Rayleigh Ritz method is used. The frequencies for the first and second mode of vibration are calculated for the trapezoidal plate having C-S-C-S edges for the different values of taper constant , thermal gradient and aspect ratio and presented in graphically form.

\section{Method of Analysis}

Let us assume that orthotropic trapezoidal plate under consideration is subjected to steady one dimensional temperature distribution $\tau$ along the length, therefore one can take $\tau$ as,

$$
\tau=\tau_{0}\left(1-\left(\xi+\frac{1}{2}\right)\right)
$$

where $\tau_{0}$ is the temperature at $\xi=-\frac{1}{2}$.

For most orthotropic materials modulus of elasticity are described as a function of temperature as,

$$
\left.\begin{array}{l}
E_{\xi}(\tau)=E_{1}(1-\gamma \tau) \\
E_{\eta}(\tau)=E_{2}(1-\gamma \tau) \\
G_{\xi \eta}(\tau)=G_{0}(1-\gamma \tau)
\end{array}\right\}
$$

Using Equation (1), Equation (2) becomes

$$
\left.\begin{array}{l}
E_{\xi}=E_{1}\left[1-\alpha\left(\frac{1}{2}-\xi\right)\right] \\
E_{\eta}=E_{2}\left[1-\alpha\left(\frac{1}{2}-\xi\right)\right] \\
G_{\xi \eta}=G_{0}\left[1-\alpha\left(\frac{1}{2}-\xi\right)\right]
\end{array}\right\}
$$

where $E_{1}, E_{2}$ and $G_{0}$ are the values of moduli at some reference temperature, i.e. $\tau=0$. 
The parabolically varying plate thickness can be express as,

$$
h(x)=h_{0} g(x)
$$

where $\mathrm{g}(\mathrm{x})$ is the thickness variation function.

The non-dimensional thickness variation function can be expressed as,

$$
g(\xi)=\left[1-(1-\beta)\left(\xi+\frac{1}{2}\right)^{2}\right], g(\xi) \geq 0
$$

With the assumption of small free vibration, the displacement function is periodic in time so it can be expressed as,

$$
w(x, y, t)=W(x, y) \sin \omega t
$$

where $\omega$ denotes the frequency of vibration.

The energy functional for the plate is,

$$
F=V-T
$$

The expression for the strain energy $\mathrm{V}$ and kinetic energy $\mathrm{T}$ in the plate is given by,

$$
\begin{gathered}
V=\frac{a b}{2} \iint\left[D_{\xi}\left(\frac{\partial^{2} W}{\partial \xi^{2}}\right)^{2}+D_{\eta}\left(\frac{\partial^{2} W}{\partial \eta^{2}}\right)^{2}\right. \\
+2 D_{1}\left(\frac{\partial^{2} W}{\partial \xi^{2}}\right)\left(\frac{\partial^{2} W}{\partial \eta^{2}}\right) \\
\left.+4 D_{\xi \eta}\left(\frac{\partial^{2} W}{\partial \xi \partial \eta}\right)^{2}\right] d \xi d \eta \\
T=\frac{a b}{2} \omega^{2} h_{0} \iint \rho g(\xi) W^{2} d \xi d \eta
\end{gathered}
$$

Expression for flexural rigidity and torsion rigidity are,

$$
\begin{aligned}
& D_{\xi}=\frac{E_{1}\left[1-\alpha\left(\frac{1}{2}-\xi\right)\right] h_{0}^{3}\left[1-(1-\beta)\left(\xi+\frac{1}{2}\right)^{2}\right]^{3}}{12\left(1-v_{\xi} v_{\eta}\right)} \\
& D_{\eta}=\frac{E_{2}\left[1-\alpha\left(\frac{1}{2}-\xi\right)\right] h_{0}^{3}\left[1-(1-\beta)\left(\xi+\frac{1}{2}\right)^{2}\right]^{3}}{12\left(1-v_{\xi} v_{\eta}\right)} \\
& D_{\xi \mu}=\frac{G_{0}\left[1-\alpha\left(\frac{1}{2}-\xi\right)\right] h_{0}^{3}\left[1-(1-\beta)\left(\xi+\frac{1}{2}\right)^{2}\right]^{3}}{12}
\end{aligned}
$$

Also

$$
D_{1}=v_{\eta} D_{\xi}=v_{\xi} D_{\eta}
$$

Introducing non-dimensional coordinate system as

$$
\xi=x / a, \eta=y / b
$$

where $a$ and $b$ are the span and width of the plate plan form as shown in Figure 1.

To apply Rayleigh Ritz technique, one must has

$$
\delta(F)=0
$$

The two term deflection function taken as,

$$
\begin{aligned}
w= & {\left[\xi+\frac{1}{2}\right]^{2}\left[\eta-\left(\frac{b-c}{2}\right) \xi+\left(\frac{b+c}{4}\right)\right] } \\
\times & {\left[\xi-\frac{1}{2}\right]^{2}\left[\eta+\left(\frac{b-c}{2}\right) \xi-\left(\frac{b+c}{4}\right)\right] } \\
\times & \times A_{1}+A_{2}\left(\xi+\frac{1}{2}\right) \\
& \times\left(\eta-\left(\frac{b-c}{2}\right) \xi+\left(\frac{b+c}{4}\right)\right)\left(\xi-\frac{1}{2}\right) \\
& \left.\times\left(\eta+\left(\frac{b-c}{2}\right) \xi-\left(\frac{b+c}{4}\right)\right)\right]
\end{aligned}
$$

where $A_{1}$ and $A_{2}$ are constants.

The orthotropic plate with symmetric parabolically varying thickness and symmetric trapezoidal plan form is taken. The thickness $\mathrm{h}_{0}$ is along the edge $\mathrm{x}=-\mathrm{a} / 2$ and $\beta h_{0}$ is the plate thickness at the edge $\mathrm{x}=\mathrm{a} / 2$, where $\beta$ is the thickness variation ratio.

Two edges of the plates are clamped and two are simply supported i.e. all the four degree of freedoms of the nodes to the side faces of the plates are constrained as shown in the Figure 1.

Here limit of $\eta$ is $-\frac{b+c}{4 b}-\frac{(c-b) a}{2 b} \xi$ to $\frac{b+c}{4 b}+\frac{(c-b) a}{2 b} \xi$ and limit of $\xi$ is -0.5 to 0.5 .

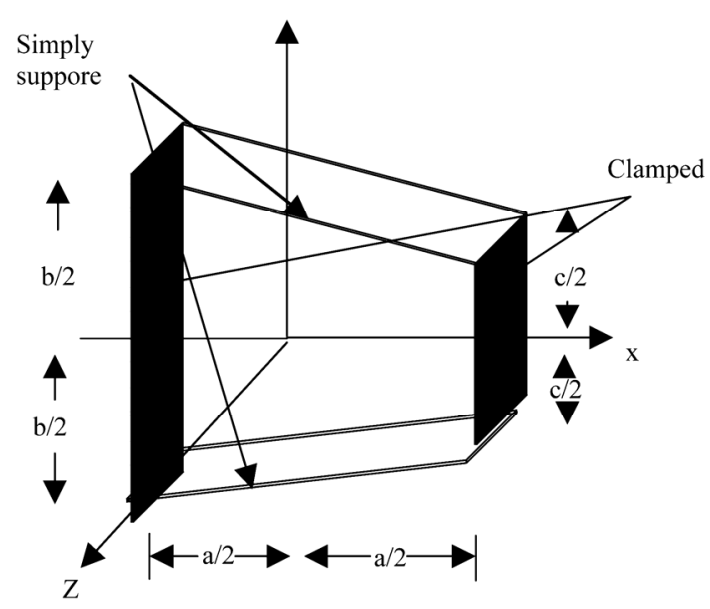

Figure 1. The geometry of symmetry trapezoidal thin plate. 
Assume that the density varies parabolically in $\mathrm{x}$ direction.

$$
\rho=\rho_{o}\left[1-\left(1-\alpha_{1}\right)(x+0.5)^{2}\right]
$$

where $\alpha_{1}$ is the non homogeneity of the plate.

Substitute Equations (5), (9) to (11) and (13) in Equations (7) and (8), one has

$$
\begin{aligned}
& V=\frac{h_{0}^{3} a b E_{1}}{24 a^{4}\left(1-v_{\xi} v_{\eta}\right)} \iint\left[\left[1-\alpha\left(\frac{1}{2}-\xi\right)\right]\right. \\
& \times\left[1-(1-\beta)\left(\xi+\frac{1}{2}\right)^{2}\right]^{3}\left(\frac{\partial^{2} W}{\partial \xi^{2}}\right)^{2} d \xi d \eta \\
& +\frac{h_{0}^{3} a b E_{2}}{24 b^{4}\left(1-v_{\xi} v_{\eta}\right)} \iint\left[1-\alpha\left(\frac{1}{2}-\xi\right)\right] \\
& \times\left[1-(1-\beta)\left(\xi+\frac{1}{2}\right)^{2}\right]^{3}\left(\frac{\partial^{2} W}{\partial \eta^{2}}\right)^{2} d \xi d \eta \\
& +\frac{h_{0}^{3} E_{1} v_{\eta}}{6 a^{2} b^{2}\left(1-v_{\xi} v_{\eta}\right)} \iint\left[1-\alpha\left(\frac{1}{2}-\xi\right)\right] \\
& \times\left[1-(1-\beta)\left(\xi+\frac{1}{2}\right)^{2}\right]^{3}\left(\frac{\partial^{2} W}{\partial \xi^{2}}\right)\left(\frac{\partial^{2} W}{\partial \eta^{2}}\right) d \xi d \eta \\
& +\frac{h_{0}^{3} G_{0}}{3 a^{2} b^{2}} \iint\left[1-\alpha\left(\frac{1}{2}-\xi\right)\right] \\
& \left.\times\left[1-(1-\beta)\left(\xi+\frac{1}{2}\right)^{2}\right]^{3}\left(\frac{\partial^{2} W}{\partial \xi \partial \eta}\right)^{2} d \xi d \eta\right] \\
& {\left[\int\left[1-\left(1-\alpha_{1}\right)(x+0.5)^{2}\right]\left[1-(1-\beta)\left(\xi+\frac{1}{2}\right)^{2}\right] W^{2} d \xi d \eta\right]}
\end{aligned}
$$

Using Equations (14) and (15) in (6) we have,

$$
\delta\left(V_{1}-\lambda^{2} T_{1}\right)=0
$$

Where

$$
\begin{aligned}
V_{1}= & \iint\left[1-\alpha\left(\frac{1}{2}-\xi\right)\right]\left[1-(1-\beta)\left(\xi+\frac{1}{2}\right)^{2}\right]^{3} \\
& \times\left(\left(\frac{\partial^{2} W}{\partial \xi^{2}}\right)^{2}+\frac{E_{2}}{E_{1}}\left(\frac{\partial^{2} W}{\partial \eta^{2}}\right)^{2}\right. \\
& +2 v_{\xi} \frac{E_{2}}{E_{1}}\left(\frac{\partial^{2} W}{\partial \xi^{2}}\right)\left(\frac{\partial^{2} W}{\partial \eta^{2}}\right) \\
& \left.+4 \frac{G_{0}\left(1-v_{\xi} v_{\eta}\right)}{E_{1}}\left(\frac{\partial^{2} W}{\partial \xi \partial \eta}\right)^{2}\right) d \xi d \eta
\end{aligned}
$$

$$
\begin{aligned}
T_{1}= & \iint\left[1-\left(1-\alpha_{1}\right)(x+0.5)^{2}\right] \\
& \times\left[1-(1-\beta)\left(\xi+\frac{1}{2}\right)^{2}\right] W^{2} d \xi d \eta
\end{aligned}
$$

and $\lambda^{2}=\frac{12 \rho_{0} \omega^{2} a^{5}\left(1-v_{\xi} v_{\eta}\right)}{E_{1} h_{0}^{2}}$ is a frequency parameter.

Equation (16) contains two unknown constants $A_{1}$ and $\mathrm{A}_{2}$ which can be evaluated as

$$
\frac{\partial\left(V_{1}-\lambda^{2} T_{1}\right)}{\partial A_{n}}=0, n=1,2
$$

The Equation (17) simplifies to the form

$$
c_{n 1} A_{1}+c_{n 2} A_{2}=0, n=1,2
$$

where $c_{n 1}$ and $c_{n 2}(n=1,2)$ involves parametric constants and frequency parameter.

For a non-zero solution, the coefficient matrix of Equation (18) must be zero. In this way the frequency equation must comes out to be

$$
\left|\begin{array}{ll}
c_{11} & c_{12} \\
C_{21} & c_{22}
\end{array}\right|=0
$$

From Equation (19) one can obtain a quadratic equation in $\lambda^{2}$ from which two values of $\lambda^{2}$ can be found.

\section{Results and Discussion}

Numerical results are obtained for the first two modes of vibrations for transverse vibration of non-homogeneous orthotropic trapezoidal plate of parabolically varying thickness with thermal gradient effect and non-homo- geneity effect for different values of aspect ratios. Frequency (19) is quadratic in $\lambda^{2}$, so it will give two roots.

The parameters for orthotropic material have been taken as [3]:

$$
\frac{E_{2}}{E_{1}}=0.32, v_{\xi} \frac{E_{2}}{E_{1}}=0.04, \frac{G_{0}}{E_{1}}\left(1-v_{\xi} v_{\eta}\right)=0.09
$$

These results are plotted in Figures 2-9.

A study will now be presented for a set of trapezoidal plate configurations by varying $\mathrm{c} / \mathrm{b}$ and $\mathrm{a} / \mathrm{b}$ values.

In Figure 2, results have been displayed for the following values:

$$
\begin{aligned}
& a / b=1.0, c / b=0.5, \\
& \alpha=0.0,0.2,0.4,0.6,0.8,1.0 \\
& \beta=0.0,0.2 \\
& \alpha_{1}=0.0,0.2
\end{aligned}
$$

It can be concluded that with the increase in thermal gradient, frequency decreases for both the modes.

Result have been displayed for the following values $\mathrm{a} / \mathrm{b}=1.0, \mathrm{c} / \mathrm{b}=0.5$ 


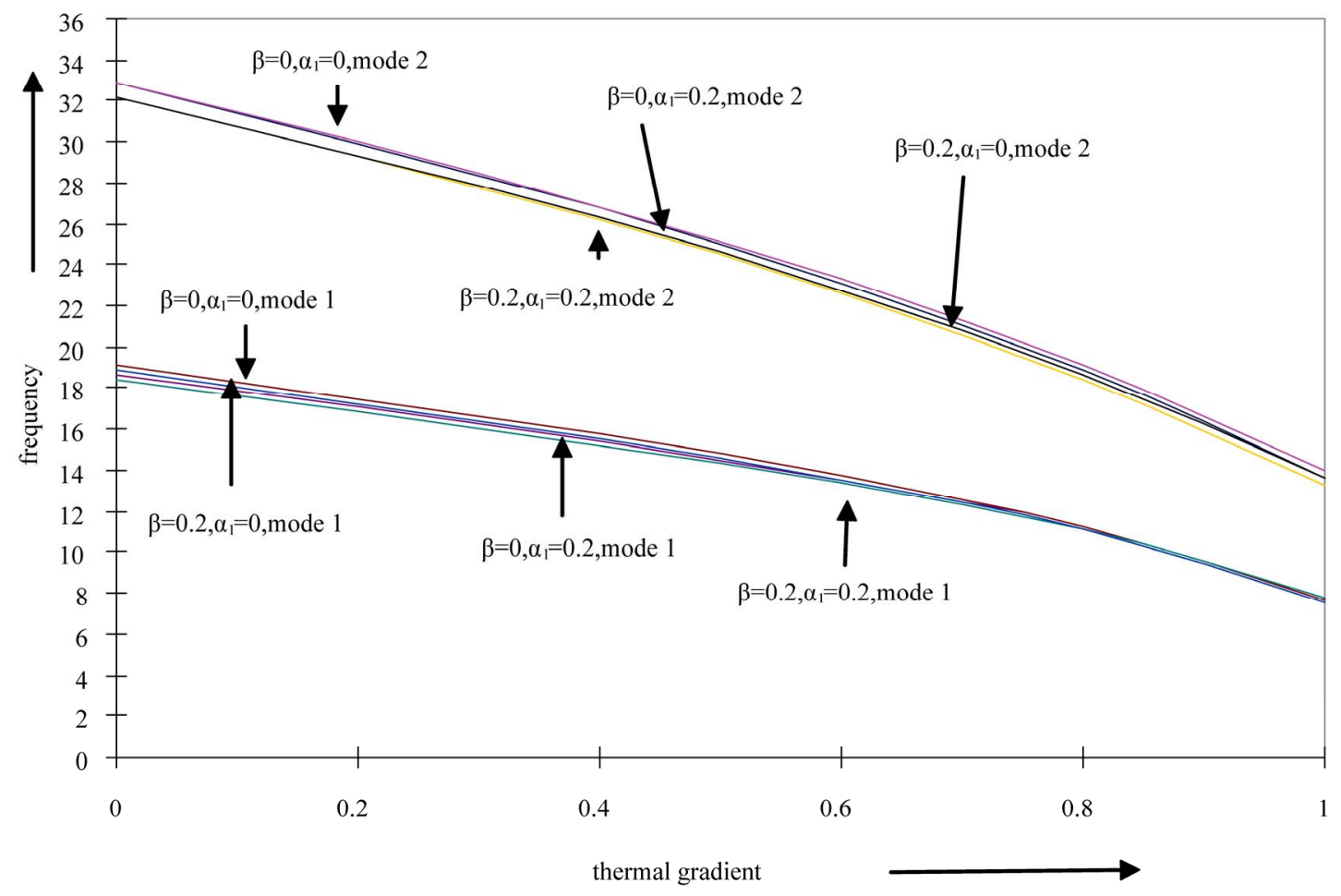

Figure 2. Value of frequency parameter for orthotropic trapezoidal plate for different values of thermal gradient and fixed values for $\mathbf{a} / \mathrm{b}=1, \mathrm{c} / \mathrm{b}=\mathbf{0 . 5}$.

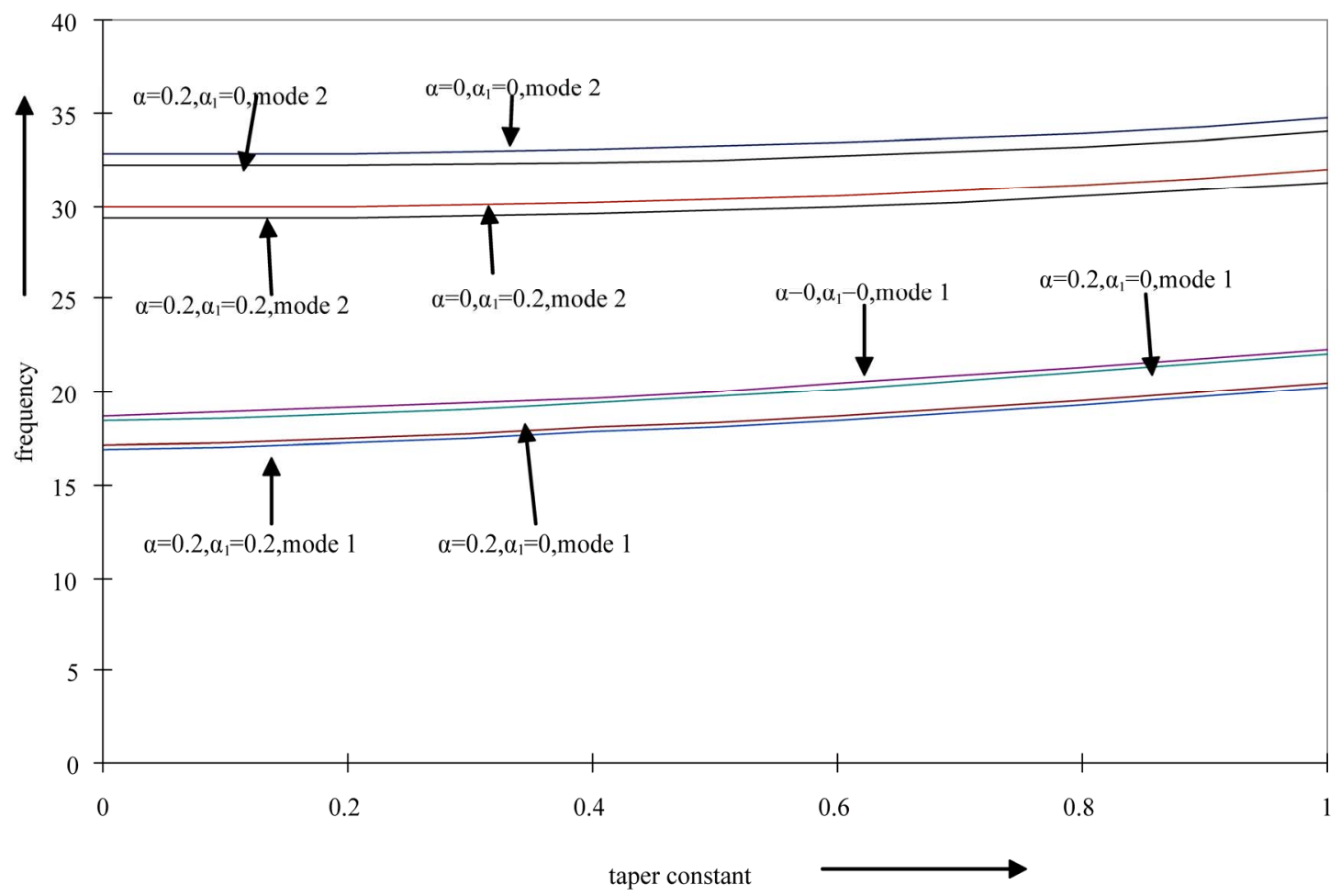

Figure 3. Value of frequency parameter for orthotropic trapezoidal plate for different values of taper constant and fixed values for $\mathrm{a} / \mathrm{b}=\mathbf{1 ,} \mathrm{c} / \mathrm{b}=\mathbf{0 . 5}$. 


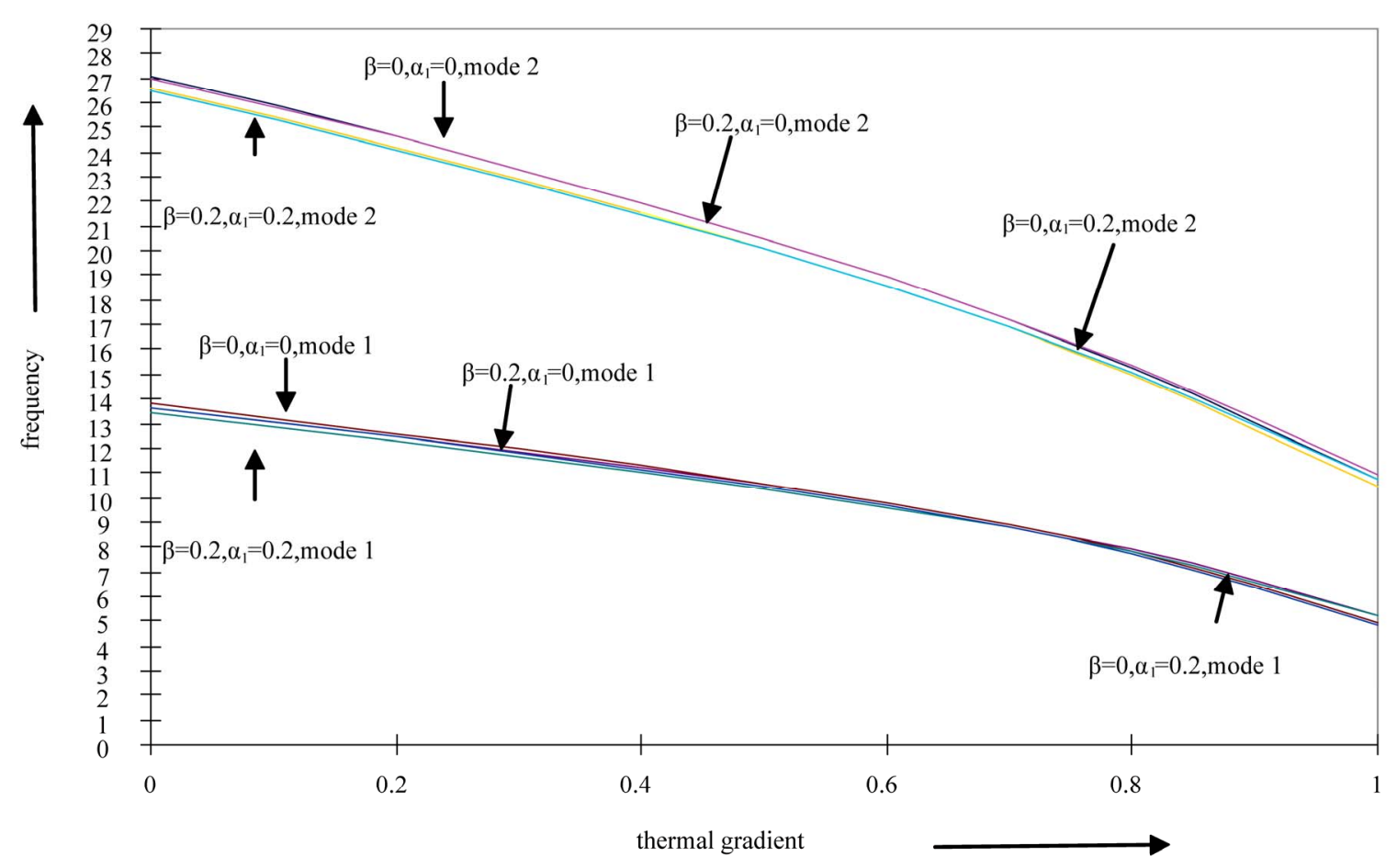

Figure 4. Value of frequency parameter for orthotropic trapezoidal plate for different values of thermal gradient and fixed values for $\mathbf{a} / \mathbf{b}=\mathbf{0 . 7 5}, \mathbf{c} / \mathbf{b}=\mathbf{0 . 5}$.

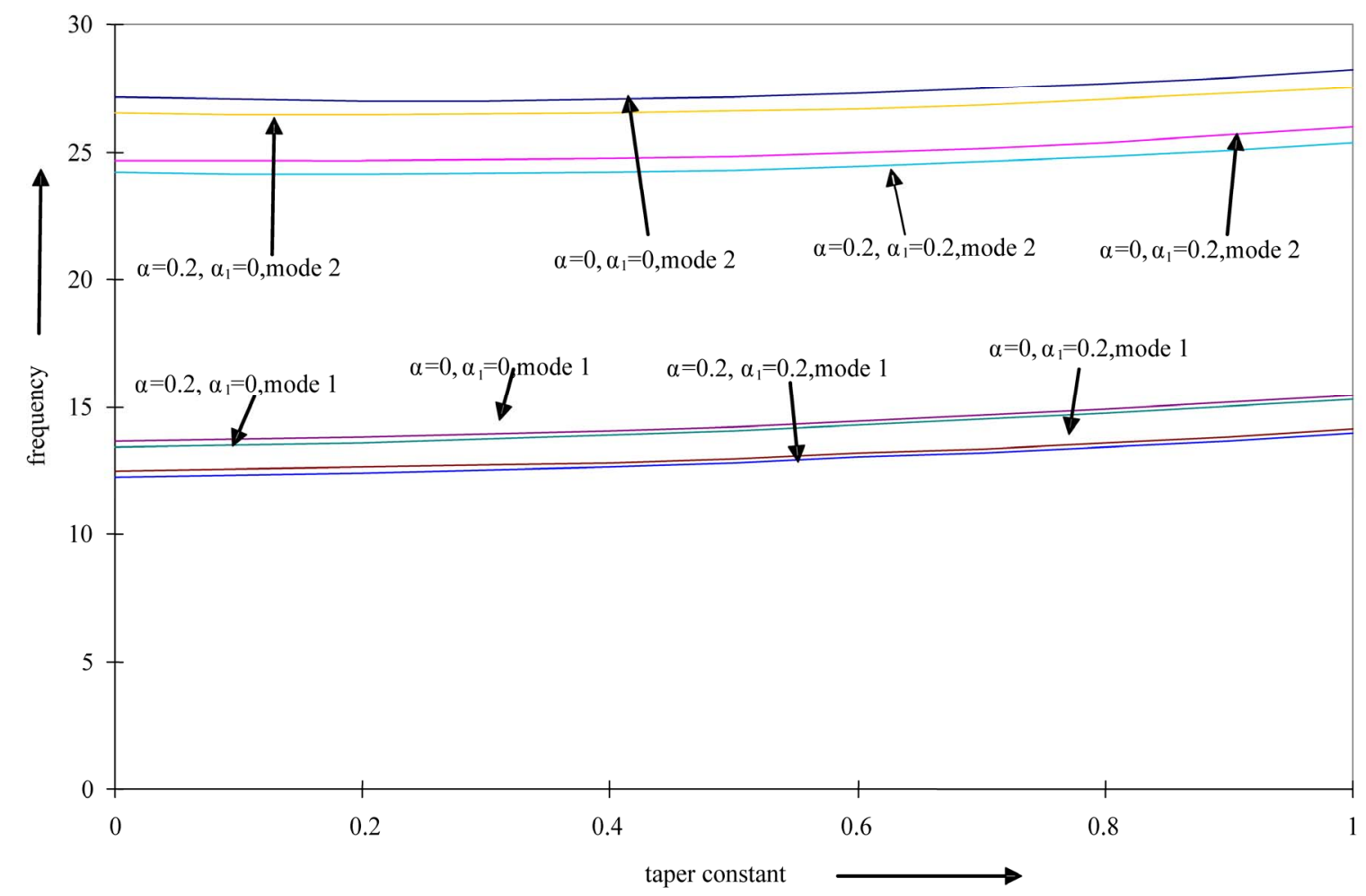

Figure 5. Value of frequency parameter for orthotropic trapezoidal plate for different values of taper constant and fixed values for $\mathbf{a} / \mathbf{b}=\mathbf{0 . 7 5}, \mathbf{c} / \mathbf{b}=\mathbf{0 . 5}$. 


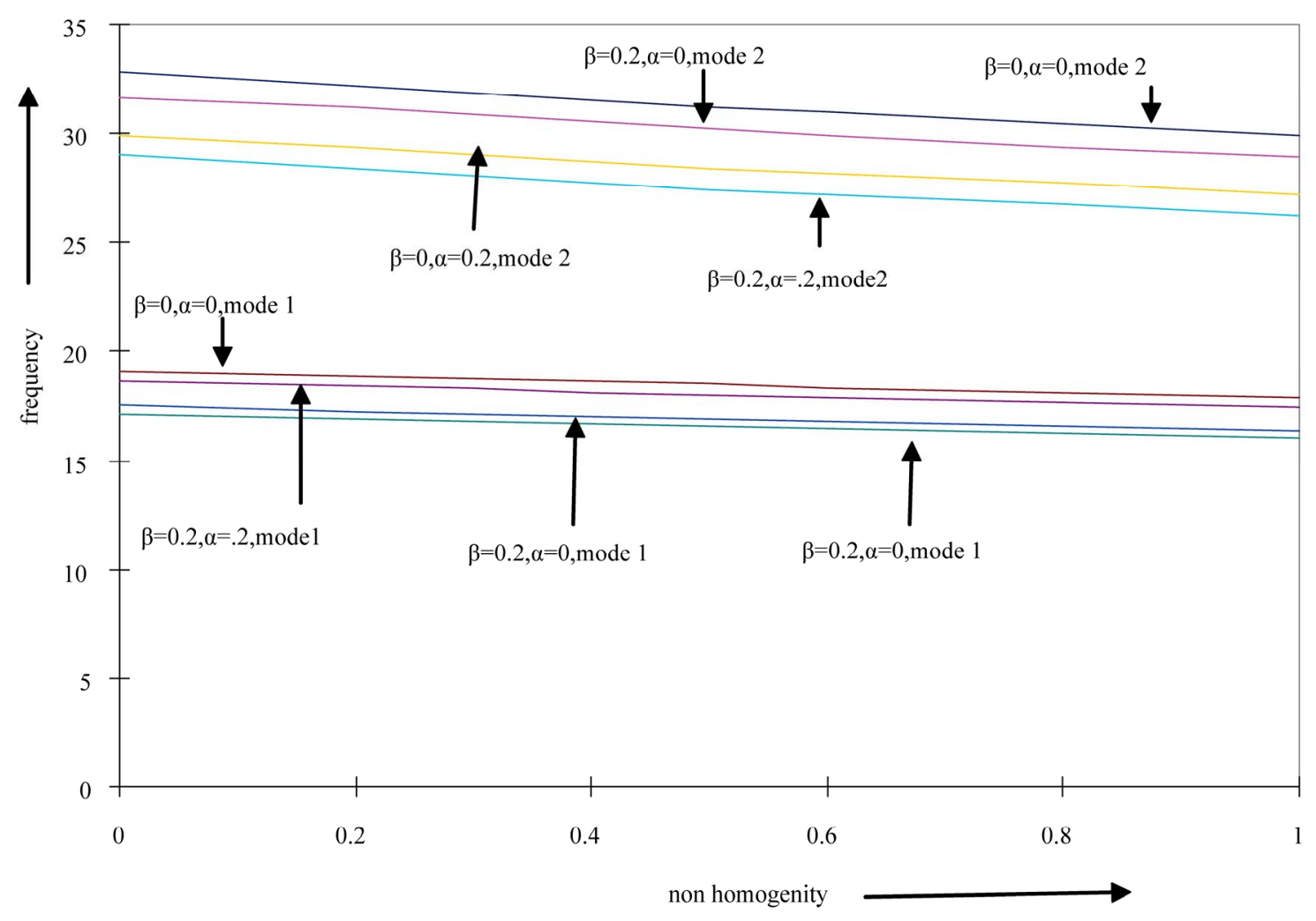

Figure 6. Value of frequency parameter for orthotropic trapezoidal plate for different values of non homogeneity and fixed values for $\mathbf{a} / \mathrm{b}=1.0, \mathrm{c} / \mathrm{b}=\mathbf{0 . 5}$.

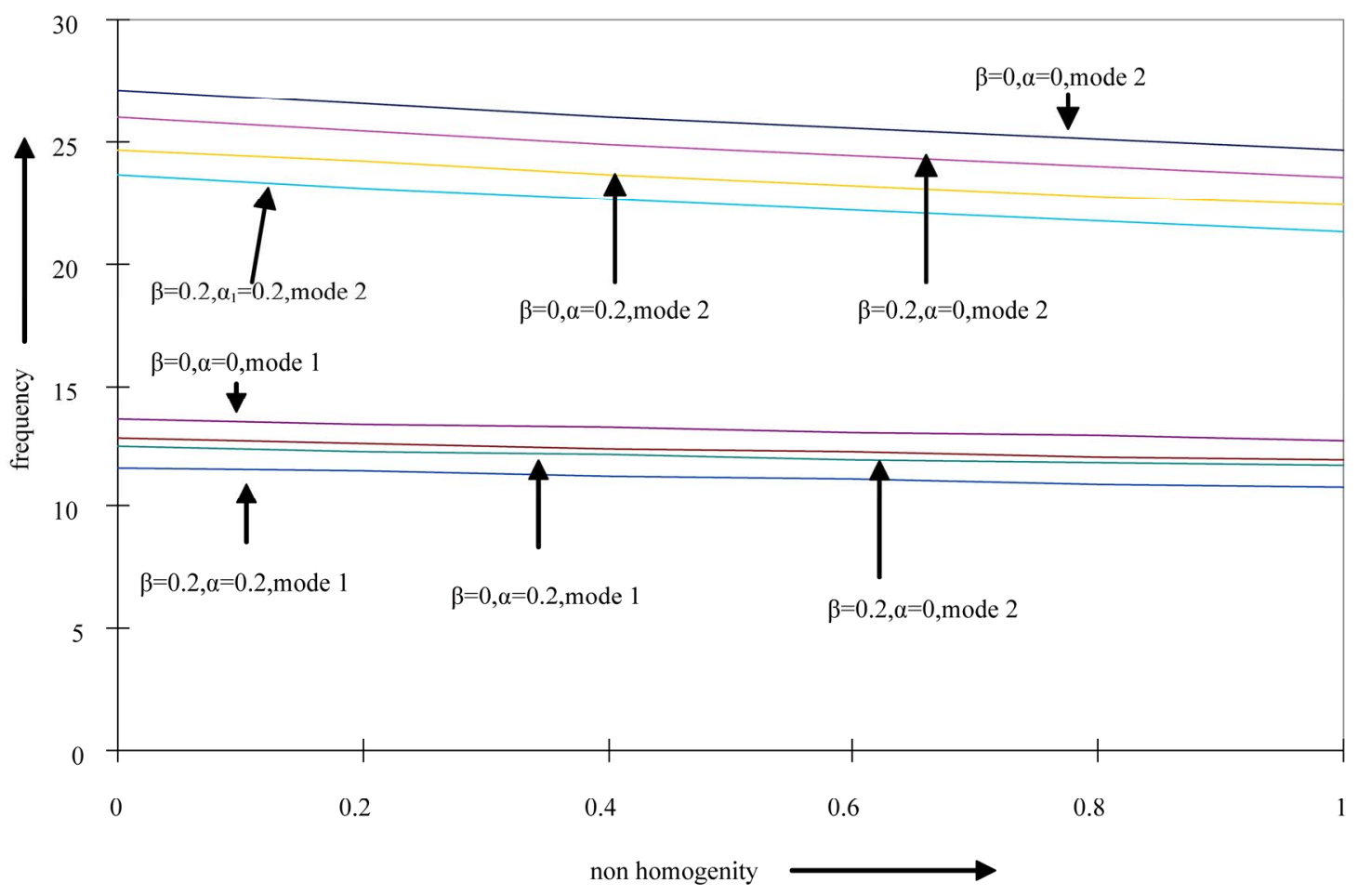

Figure 7. Value of frequency parameter for orthotropic trapezoidal plate for different values of non homogeneity and fixed values for $\mathbf{a} / \mathbf{b}=0.75, \mathbf{c} / \mathbf{b}=0.5$. 


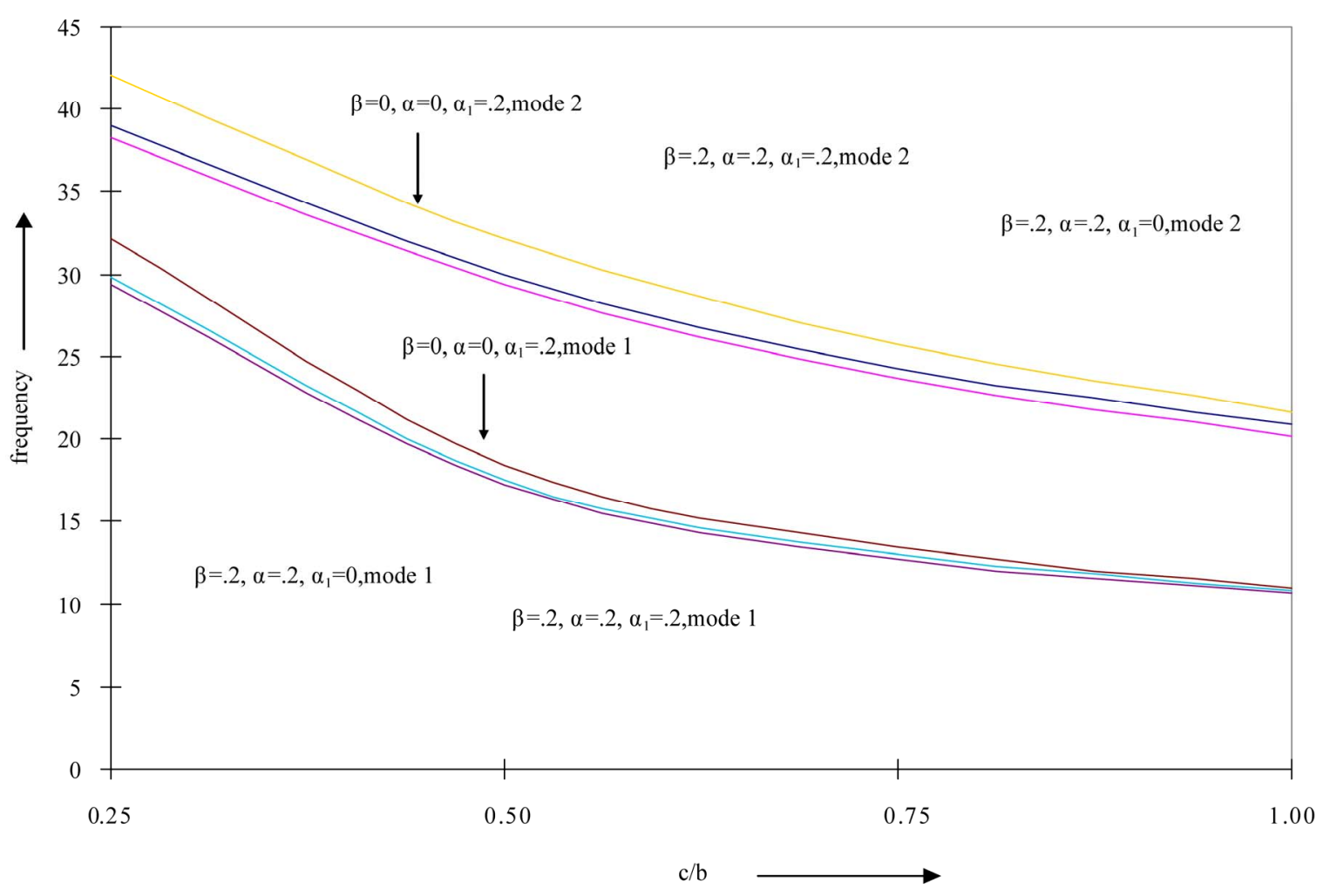

Figure 8. Value of frequency parameter for orthotropic trapezoidal plate for different values of $c / b$ and fixed values for $a / b=1$.

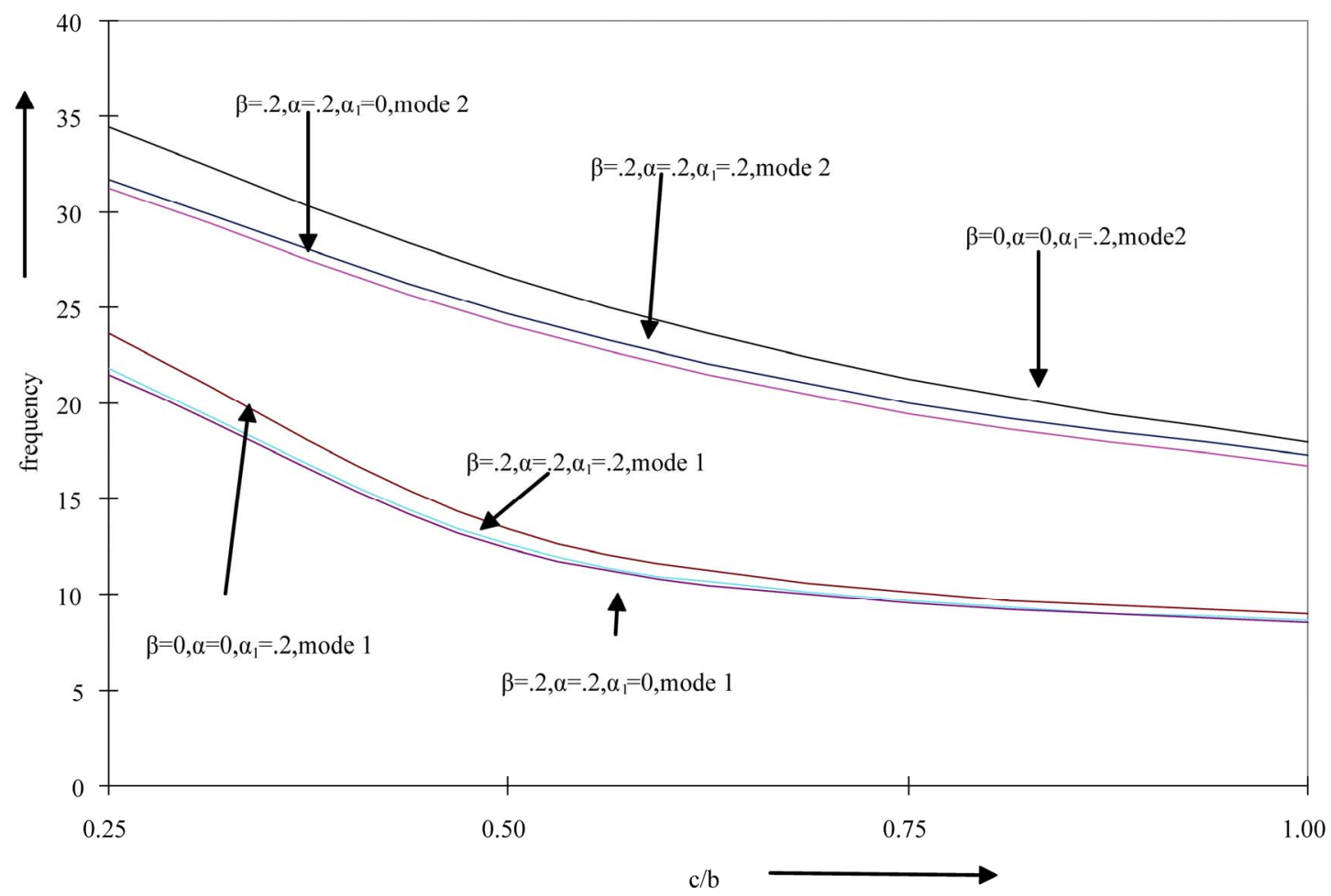

Figure 9. Value of frequency parameter for orthotropic trapezoidal plate for different values of $c / b$ and fixed values for $\mathbf{a} / \mathbf{b}=\mathbf{0 . 7 5}$. 
$\beta=0.0,0.2,0.4,0.6,0.8,1.0$

$\alpha=0.0,0.2$

$\alpha_{1}=0.0,0.2$

In Figure 3. It can be concluded that with the increase in taper constant, frequency increases for both the modes.

In Figure 4, results have been displayed for the following values:

$\mathrm{a} / \mathrm{b}=0.75, \mathrm{c} / \mathrm{b}=0.5$

$\alpha=0.0,0.2,0.4,0.6,0.8,1.0$

$\beta=0.0,0.2$

$\alpha_{1}=0.0,0.2$

It can be concluded that with the increase in thermal gradient, frequency decreases for both the modes.

Figure 5 show the results for the following values:

$\mathrm{a} / \mathrm{b}=0.75, \mathrm{c} / \mathrm{b}=0.5$

$\beta=0.0,0.2,0.4,0.6,0.8,1.0$

$\alpha=0.0,0.2$

$\alpha_{1}=0.0,0.2$

and it can be concluded that with the increase in taper constant, frequency increases for both the modes.

In Figure 6, results have been displayed for the following values:

$\mathrm{a} / \mathrm{b}=1.0, \mathrm{c} / \mathrm{b}=0.5$

$\alpha_{1}=0.0,0.2,0.4,0.6,0.8,1.0$

$\beta=0.0,0.2$

$\alpha=0.0,0.2$

It can be concluded that with the increase in non homogeneity, frequency decreases for both the modes.

In Figure 7, results have been displayed for the following values:

$\mathrm{a} / \mathrm{b}=0.75, \mathrm{c} / \mathrm{b}=0.5$

$\alpha_{1}=0.0,0.2,0.4,0.6,0.8,1.0$

$\beta=0.0,0.2$

$\alpha=0.0,0.2$

It can be concluded that with the increase in non homogeneity, frequency decreases for both the modes.

In Figure 8, results have been displayed for the following values:

$\mathrm{a} / \mathrm{b}=1.0 ; \mathrm{c} / \mathrm{b}=0.25,0.5,0.75,1.0 ;$

$\beta=0.0,0.2$;

$\alpha=0.0,0.2$

$\alpha_{1}=0.0,0.2$

It can be concluded that with the increase in the value of $\mathrm{c} / \mathrm{b}$, frequency decreases for both the modes and for all combinations of $\alpha, \alpha_{1}$ and $\beta$.

In Figure 9, results have been displayed for the following values:

$$
\begin{aligned}
& a / b=0.75 ; c / b=0.25,0.5,0.75,1.0 ; \\
& \beta=0.0,0.2 ; \\
& \alpha=0.0,0.2 \\
& \alpha_{1}=0.0,0.2
\end{aligned}
$$

It can be concluded that with the increase in the value of $\mathrm{c} / \mathrm{b}$, frequency decreases for both the modes and for all combinations of $\alpha, \alpha_{1}$ and $\beta$.

\section{Conclusions}

The paper presented a comprehensive review for the analysis of the linear vibration characteristics of orthotropic trapezoidal plates. The review covered numerical procedures by using Ritz method. The influence of various parameters as non homogeneity, taper constant, thermal gradient etc, affecting the frequency was also discussed. Accurate data has obtained by varying the length ratios $\mathrm{a} / \mathrm{b}$ and $\mathrm{c} / \mathrm{b}$. The results for orthotropic trapezoidal plates of parabolically varying thickness are verified by the literature $[3,16]$. It has been shown that the method provides accurate results.

The frequencies presented in this paper are considerable accurate in order that these should be useful in subsequent research carried out for these plates.

\section{References}

[1] A. W. Leissa, "Recent Studies in Plate Vibration 19811985 Part II, Complicating Effects," The Shock and Vibration Digest, Vol. 19, No. 3, 1987, pp. 10-24. doi 10.1177/058310248701900304

[2] A. W. Leissa, "Recent Studies in Plate Vibration: 19811985. Part Classical Theory," The Shock and Vibration Digest, Vol. 19, No. 2, 1987, pp. 11-18. doi:10.1177/ 058310248701900204

[3] A. W. Leissa, Vibration of Plates, NASA SP-160, 1969.

[4] I. Chopra and S. Durvasula, "Vibration of Simple-Supported Trapezoidal Plates. I: Symmetric Trapezoids," Journal of Sound and Vibration, Vol. 19, No. 4, 1971, pp. 379-392. doi:10.1016/0022-460X(71)90609-2

[5] P. S. Nair and S. Durvasula, "Vibration of Skew Plate," Journal of Sound Vibration, Vol. 26, No. 1, 1973, pp. 1-20. doi:10.1016/S0022-460X(73)80201-9

[6] J. S. Tomar and A. K. Gupta, "Thermal Effect on Axisymmetric Vibration of an Orthotropic Circular Plate of Variable Thickness," American Institute of Aeronautics and Astronautics, Vol. 22, No. 7, 1984, pp. 1015-1017.

[7] J. S. Tomar and A. K. Gupta, "Thermal Effect of Frequencies of an Orthotropic Rectangular Plate of Linearly Varying Thickness," Journal of Sound and Vibration, Vol. 90, No. 3, 1983, pp. 325-331. doi:10.1016/0022-460X(83) 90715-0

[8] R. M Orris and M. Petyt, "A Finite Element Study of the Vibration of Trapezoidal Plates," Journal of Sound and Vibration, Vol. 27, No. 3, 1973, 325-344. doi:10.1016/ S0022-460X(73)80349-9

[9] Y. Narita, K. Maruyama and M. Sonada "Transverse Vibration of Clamped Trapezoidal Plates Having Rectangular Orthotropy," Journal of Sound and Vibration, Vol. 85, No. 3, 1982, pp. 315-322. doi:10.1016/0022$460 \times(82) 90257-7$ 
[10] S. Mirza and M. Bijlani, "Vibration of Triangular Plates," American Institute of Aeronautics and Astronautics, Vol. 21, No. 10, 1983, pp. 1472-1475.

[11] N. S. Bhatnagar and A. K. Gupta, "Thermal Effect on Vibration of Visco Elastic Elliptic Plate of Variable Thickness," Proceedings of International Conference on Modelling and Simulation, Melbourn, 1987, pp. 424-429.

[12] H. T. Sahba, "Transverse Free Vibration of Fully Clamped Symmetrical Trapezoidal Plates," Journal of Sound and Vibration, Vol. 126, No. 2, 1988, pp. 237-247. doi: 10.1016/0022-460X(88)90238-6

[13] P. Laura, R. Gutierrez and R. Bhat, "Transverse Vibrations of a Trapezoidal Cantilever Plate of Various Thickness," AIAA, Vol. 27, No. 7, 1989, pp. 921-922. doi: $10.2514 / 3.10201$

[14] K. M. Liew and K. Y. Lam, "A Rayleigh-Ritz Approach to Transverse Vibration of Isotropic and Anisotropic Trapezoidal Plates Using Orthogonal Plate Functions," International Journal of Solids and Structures, Vol. 27, No. 2, 1991, pp. 189-203. doi:10.1016/0020-7683(91) 90228-8

[15] K. M. Liew, "Variable of Symmetric Laminated Cantilever Trapezoidal Composite Plates," Journal of Mechanical Sciences, Vol. 34, No. 4, 1992, pp. 299-308. doi: 10.1016/0020-7403(92)90037-H

[16] K. M. Liew and M. K. Lim, "Transverse Vibration of Trapezoidal Plates of Variable Thickness: Symmetric Trapezoids," Journal of Sound and Vibration, Vol. 165, No. 1, 1993, pp. 45-67. doi:10.1006/jsvi.1993.1242

[17] M. S. Qatu, N. A. Jaber and A. W. Leissa, "Natural Frequencies for Completely Free Trapezoidal Plates," Journal of Sound and Vibration, Vol. 167, No. 1, 1993, pp. 183-191. doi:10.1006/jsvi.1993.1328

[18] C. W. Lim and K. M. Liew, "Vibration of Pretwisted Cantilever Trapezoidal Symmetric Laminates," Acta Mechanica, Vol. 111, No. 3-4, 1995, pp. 193-208. doi: 10.

\section{7/BF01376930}

[19] T. Sakiyama and M. Hung, "Free Vibration Analysis of Right Triangular Plates with Variable Thickness," Journal of Sound and Vibration, Vol. 234, No. 5, 2000, pp. 841-858. doi:10.1006/jsvi.2000.2903

[20] R. Lal, "Transverse Vibration of Orthotropic Non Uniform Rectangular Plate with Continuously Varying Density," Indian Journal of Pure and Applied Mathematics, Vol. 34, No. 4, 2003, pp. 587-606.

[21] A. W. Leissa, "The Historical Bases of the Rayleigh and Ritz Methods," Journal of Sound and Vibration, Vol. 287, No. 4-5, 2005, pp. 961-978. doi:10.1016/j.jsv.2004.12.021

[22] C.-H. Huang, C.-H. Hsu and Y.-K. Lin, "Experimental and Numerical Investigations for the Free Vibration of Cantilever Trapezoidal Plates," Journal of the Chinese Institute of Engineers, Vol. 29, No. 5, 2006, pp. 863-872.

[23] A. K. Gupta, Johri Tripti and R. P. Vats, "Thermal Effect on Vibration of Non Homogeneous Orthotropic Rectangular Plate Having Bi-directional Parabolically Varying Thickness," Proceedings of International Conference on Engineering and Computer Science, San Francisco, 2007, pp. 784-787.

[24] A. K. Gupta, Johri Tripti and R. P. Vats, "Thermal Gradient Effect on Vibration of a Non-Homogeneous Orthotropic Rectangular Plate Having Bi-direction Linearly Thickness Variation," Meccanica, Vol. 45, No. 3, 2010, pp. 393-400. doi:10.1007/s11012-009-9258-3

[25] G. Karami, S. A. Shahpari and P. Malekzadeh, "DQM Analysis of Skewed and Trapezoidal Laminated Plates," Computer Structures, Vol. 59, No. 3, 2003, pp. 393-402. doi: 10.1016/S0263-8223(02)00188-5

[26] A. K. Gupta and S. Sharma, "Thermally Induced Vibration of Orthotropic Trapezoidal Plate of Linearly Varying Thickness," Journal of Vibration and Control (Accepted for publication). 